\title{
1 Proton-pumping rhodopsins in marine diatoms
}

2

3 Susumu Yoshizawa ${ }^{\mathrm{a}, \mathrm{b}, \mathrm{c}}{ }^{\dagger}$, Tomonori Azuma ${ }^{\mathrm{d}}$, Keiichi Kojima ${ }^{\mathrm{e}, \mathrm{f}}$, Keisuke Inomura ${ }^{\mathrm{g}}$,

4 Masumi Hasegawa ${ }^{\mathrm{a}, \mathrm{b}}$, Yosuke Nishimura ${ }^{\mathrm{a}}$, Masuzu Kikuchi ${ }^{\mathrm{e}}$, Gabrielle Armin ${ }^{\mathrm{g}}$, Hideaki

5 Miyashita $^{\mathrm{d}}$, Kentaro Ifuku ${ }^{\mathrm{h}}$, Takashi Yamano ${ }^{\mathrm{I}}$, Adrian Marchettij, Hideya Fukuzawa,

6 Yuki Sudo ${ }^{\mathrm{e}, \mathrm{f}}$ and Ryoma Kamikawa ${ }^{\mathrm{h}}$

7

aAtmosphere and Ocean Research Institute, The University of Tokyo, Chiba 277-8564, Japan

${ }^{\mathrm{b}}$ Graduate School of Frontier Sciences, The University of Tokyo, Chiba 277-8563, Japan ${ }^{c}$ Collaborative Research Institute for Innovative Microbiology, The University of Tokyo, Tokyo 113-8657, Japan

${ }^{\mathrm{d}}$ Graduate School of Human and Environmental Studies, Kyoto University, Kyoto 6068501, Japan

eDivision of Pharmaceutical Sciences, Okayama University, Okayama 700-8530, Japan.

${ }^{\mathrm{f}}$ Graduate School of Medicine, Dentistry and Pharmaceutical Sciences, Okayama University, Okayama 700-8530, Japan.

9 gGraduate School of Oceanography, University of Rhode Island, Narragansett, RI, USA

${ }^{\mathrm{h}}$ Graduate School of Agriculture, Kyoto University, Kyoto 606-8502, Japan

1 I'Graduate School of Biostudies, Kyoto University, Kyoto, 606-8502, Japan

2 jDepartment of Earth, Marine and Environmental Sciences, University of North

3 Carolina at Chapel Hill, Chapel Hill, North Carolina, USA

$5{ }^{\dagger}$ Corresponding author Email: yoshizawa@aori.u-tokyo.ac.jp 
Abstract

Diatoms are a major phytoplankton group responsible for about $20 \%$ of Earth's primary production. They carry out photosynthesis inside the plastid, an organelle obtained through eukaryote-eukaryote endosymbiosis. Recently, microbial rhodopsin, a photoreceptor distinct from chlorophyll-based photosystems, has been identified in certain diatoms. However, the physiological function of diatom rhodopsin is not well understood. Here we show that the diatom rhodopsin acts as a light-driven proton pump and localizes to the outermost membrane of the four membrane-bound complex plastids. Heterologous expression techniques were used to investigate the protein function and subcellular localization of diatom rhodopsin. Using model simulations, we further evaluated the physiological role of the acidic pool in the plastid produced by proton-transporting rhodopsin. Our results propose that the rhodopsin-derived acidic pool may be involved in a photosynthetic $\mathrm{CO}_{2}$-concentrating mechanism and assist $\mathrm{CO}_{2}$ fixation in diatom cells. 


\section{Introduction}

Diatoms are unicellular, photosynthetic algae found throughout aquatic environments, and are responsible for up to $20 \%$ of annual net global carbon fixation ${ }^{1}$. As their contribution to primary production in the ocean is significant, their light utilization mechanisms are essential to correctly understand marine ecosystems. Diatoms contain chlorophyll a and c and carotenoids such as fucoxanthin as photosynthetic pigments in the secondary plastids acquired by eukaryote-eukaryote endosymbiosis ${ }^{2}$. In addition, certain diatoms have recently been shown to contain microbial rhodopsin (henceforth rhodopsins), a light-harvesting antenna distinct from the chlorophyll-containing antenna for photosynthesis ${ }^{3}$. Although rhodopsin-mediated light-harvesting may somehow support the survival of these diatoms in marine environments, the physiological role of rhodopsin in diatom cells remains unclear.

Microbial rhodopsins are a large family of seven transmembrane photoreceptor proteins ${ }^{4}$. Rhodopsin has an all-trans retinal as the light-absorbing chromophore, and its protein function is triggered by light-induced isomerization of the retinal. The first microbial rhodopsin, the light-driven proton pump bacteriorhodopsin (BR), was discovered in halophilic archaea ${ }^{5}$. Although rhodopsin was initially thought to occur only in halophilic archaea inhabiting hypersaline environments, subsequent studies have shown that the rhodopsin gene is widely distributed in all three domains of life $\mathrm{f}^{6,7}$. Rhodopsins can be classified based on their functions into light-driven ion pumps, lightactivated signal transducers, and light-gated ion channels. The two former functional types of rhodopsin have so far been found in prokaryotes ${ }^{8}$. The rhodopsins in prokaryotes, regardless of function, are localized to the plasma membrane in which they operate. For example, proton-pumping rhodopsins export protons from the cytosol across the plasma membrane to convert light energy into a proton motive force $(\mathrm{PMF})^{9}$. The PMF induced by rhodopsin ion transport is utilized by various physiological functions such as ATP synthesis, substrate uptake, and flagellar movement. 

pumps and light-gated ion channels have been reported ${ }^{8,10}$. Light-gated ion channels are well studied; they exclusively localize in the eyespot of phytoplankton and are involved in phototaxis ${ }^{11}$. The other type of rhodopsin in eukaryotes, light-driven ion-pumping rhodopsins have been found in a number of organisms belonging to both photoautotrophic and heterotrophic protists ${ }^{3,12}$. Since the intracellular membrane structure of eukaryotic cells is more complex than that of prokaryotes, containing a variety of organelles, even light-driven ion-pumping rhodopsins may have distinct physiological roles depending on their subcellular localization ${ }^{12}$. However, due to the difficulty in determining the exact localization of rhodopsins in eukaryotic cells, most rhodopsins are not even known for their subcellular localization.

In this study, to clarify the physiological function of rhodopsin in a marine pennate diatom, we investigated the phylogeny, protein function, spectroscopic characteristics, and subcellular localization of rhodopsin from a member of the genus Pseudo-nitzschia. Heterologous expression techniques were used to analyze the protein function and spectroscopic features. The expression eGFP-fusion rhodopsin also revealed the subcellular localization in the model diatom (Phaeodactylum tricornutum). Furthermore, a model-based analysis was performed to evaluate the impacts of the potential roles of the rhodopsin for cellular biology.

\section{Results and Discussion}

\section{Rhodopsin sequences and phylogenetic analysis}

We performed phylogenetic analysis using the rhodopsin (named PngR, accession no. AJA37445.1) of the diatom Pseudo-nitzschia granii and the microbial rhodopsin sequences reported to date ${ }^{3}$. This phylogenetic tree revealed that PngR is not included in the proteorhodopsin (PR) clade commonly found in the ocean but belongs to the Xanthorhodopsin (XR) -like rhodopsin (XLR) clade, which is presumed to have an outward proton transporting function (Fig. 1 and Extended Data Fig. 1). The 
comparison of motif sequences necessary for ion transport showed that the amino acids in the putative proton donor and acceptor sites of XR and PR were conserved in PngR, suggesting that PngR functions as an outward proton pump (Extended Data Fig. 2). Furthermore, the homology search for rhodopsin sequence in the XLR clade from Marine Microbial Eukaryote Transcriptome Sequencing Project (MMETSP) revealed that not only diatoms (Ochrophyta, Stramenopiles), but also dinoflagellates (Dinophyceae, Alveolata) and haptophytes have rhodopsin genes in the same XLR clade (Supporting Information Table S1). These results indicate that rhodopsin of the XLR clade is widely distributed among the major phytoplankton groups, which are important primary producers in the ocean.

\section{Function and spectroscopic features of Diatom rhodopsin.}

To characterize the function of the PngR, we heterologously expressed the synthesized rhodopsin gene in Escherichia coli cells. A light-induced decrease in $\mathrm{pH}$ was observed in the suspension of the E. coli cells expressing the PngR, and this decrease was almost completely abolished in the presence of the protonophore carbonyl cyanide Mchlorophenylhydrazone (CCCP) (Fig. 2A). The observed pH changes clearly showed that PngR exports protons from the cytoplasmic side across the plasma membrane.

Next, we examined the spectroscopic characteristics of PngR using recombinant protein purified from $E$. coli. The absorption maximum of PngR was located at $511 \mathrm{~nm}$ (Fig. 2B), which was explicitly shorter than that of XR (565 nm) and GR (Gloeobacter rhodopsin $541 \mathrm{~nm}$ ) in the XLR clade ${ }^{13}$. It is noteworthy that while $P$. granii is a marine species, while XR and GR are both distributed in terrestrial freshwater organisms. Thus, our observations are consistent with the shorter wavelength of the absorption maximum of rhodopsin in marine environments than in the terrestrial environment ${ }^{14}$, indicating that PngR is well adapted to the light conditions in the ocean. We then examined the retinal configuration in PngR by high-performance liquid chromatography. In both in light- and dark-adapted samples, the isomeric state of 
retinal was predominantly all-trans (Extended Data Fig. 3), which was similar to the isomeric state of retinal in prokaryotic GR in XLR clade but different from that in $\mathrm{BR}^{15}$. Since the $\mathrm{p} K_{\mathrm{a}}$ value of the proton acceptor residue (Asp85 in $\left.\mathrm{BR}\right)$ is an indicator of the efficiency of proton transport by rhodopsin, we estimated the $\mathrm{p} K_{\mathrm{a}}$ values of the putative proton acceptor in PngR (Asp91) by pH titration experiment (Extended Data Fig. 4). This experiment estimated that the $\mathrm{pKa}$ of this residue acceptor is about 5.0, indicating that the proton acceptor of PngR works well in marine and intracellular environments. Furthermore, the photochemical reactions that proceed behind the ion-transportation mechanism of PngR were examined by flash-photolysis analysis (Extended Data Fig. 5). All photocycle of PngR were completed in about $300 \mathrm{~ms}$, suggesting that the cycle is fast enough to transport protons in a physiologically significant time scale. Detailed results and discussion of flash-photolysis analysis are described in the supplementary information.

\section{Subcellular localization of PngR in a model diatom.}

The PngR sequence bears neither apparent N-terminal extension nor detectable Nterminal signal peptide, and thus in silico analyses are not able to predict a possible subcellular localization of PngR. To identify the subcellular localization of PngR, a Cterminal eGFP-fusion PngR was expressed in the model diatom Phaeodactylum tricornutum, which can be transformed by electroporation and is often used for heterologous expression analysis ${ }^{16,17}$. The transformed $P$. tricornutum cell was observed with differential interface contrast and epifluorescent microscopes (Fig. 3A). From these microscopic observations, we examined the morphology of $P$. tricornutum and the

150 localization of recombinant PngR, nucleus, chlorophyll and other cellular organelles in multiple cells (Extended Data Fig. 6 and 7). The fluorescence signal of PngR:eGFP transformant was most likely localized in the periphery of chlorophyll fluorescence and DAPI signals, corresponding to the outermost plastid membrane, called the chloroplast endoplasmic reticulum membrane (CERM), which is physically connected to the 
nuclear membrane (Fig. 3A). A few cells also exhibited GFP signals within vacuolar membranes in addition to CERM (Extended Data Fig. 7). Insertion of the complete sequence of the PngR:eGFP gene in the transformant DNA was confirmed by PCR followed by the Sanger sequencing.

Given the above heterologous expression experiment and microscopic observation, we expect that PngRs primarily localize to the outermost membrane of the plastid, although to a lesser extent, may also be found in the vacuolar membrane, which may be a function of other factors such as cell growth conditions. In addition, PngR should be oriented in the direction that the inside of the CERM is regarded as the outside of an analogous prokaryotic cell. These results imply that the light-driven proton transport by PngR could acidify the inner region of the CERM (Fig. 3B). If correct, what physiological role does the acidification of this region have in diatoms? The electrochemical gradient formed by rhodopsin could be a driving force for various secondary transport processes. Alternatively, since the primary purpose of plastids is photosynthesis, the formation of acidic pools is likely to be somehow related to photosynthesis. One possibility is that the appearance of this acidic pool influences carbon fixation, since in the carbonate system, acidification favors a higher concentration of $\mathrm{CO}_{2}$ relative to the other dominant carbonate species in seawater $\left(\mathrm{HCO}_{3}{ }^{-}\right.$and $\left.\mathrm{CO}_{3}{ }^{2-}\right)$.

In general, under weakly alkaline conditions in the ocean, most of the dissolved inorganic carbon (DIC) is present in the form of $\mathrm{HCO}_{3}{ }^{-}$, and only approximately $1 \%$ is present in the form of $\mathrm{CO}_{2}$. However, ribulose-1,5-bisphosphate carboxylase/oxygenase (RuBisCO) localized in the stroma can only react with inorganic carbon (Ci) in the form of $\mathrm{CO}_{2}$, but not of $\mathrm{HCO}_{3}{ }^{-}$. The RuBisCO enzyme in diatoms has a low affinity even for $\mathrm{CO}_{2}\left(\mathrm{Km}\right.$ of $25 \sim 68 \mu \mathrm{M}$ while $\mathrm{CO}_{2} \mathrm{aq}$ in the ocean is about $10 \mu \mathrm{M}$ at $\left.25^{\circ} \mathrm{C}\right)$ and thus requires concentrated $\mathrm{CO}_{2}$ for efficient fixation at the site of $\mathrm{RuBisCO}$. In other words, the ocean has always been a $\mathrm{CO}_{2}$-limited environment for most phytoplankton ${ }^{18}$. Consequently, due to membrane impermeability of $\mathrm{HCO}_{3}{ }^{-}$, phytoplankton have 

to the site of $\mathrm{RuBisCO}$ by placing $\mathrm{HCO}_{3}{ }^{-}$transporters in appropriate membranes and carbonic anhydrase (CA) in these compartments, the latter of which catalyzes the rapid interconversion between $\mathrm{HCO}_{3}{ }^{-}$and $\mathrm{CO}_{2}$. This interconversion can be facilitated if the balance of the carbonate system is biased toward $\mathrm{CO}_{2}$ with acidification. $\mathrm{RuBisCO}$ is located in the stroma surrounded by three or four membranes, it is necessary to localize $\mathrm{HCO}_{3}{ }^{-}$transporters in each membrane ${ }^{19}$. Diatoms that possess four membrane-bound plastids are known to have a $\mathrm{HCO}_{3}{ }^{-}$transporter (e.g., SLC4) present in the plasma membrane ${ }^{16}$, but it is not well understood how $\mathrm{CO}_{2}$ is concentrated in close proximity to $\mathrm{RuBisCO}$ in the stroma ${ }^{20}$. In sharp contrast to the transporter-based mechanism, if the equilibrium of $\mathrm{Ci}$ can be shifted toward $\mathrm{CO}_{2}$ by acidifying the inner region of the outermost membrane, it may be possible to transport $\mathrm{CO}_{2}$ to $\mathrm{RuBisCO}$ in a membrane-permeable form through gas diffusion. However, since it is difficult to directly investigate the role of membrane protein in eukaryotic microbial organelles, the combination of observation and model simulation is a powerful approach ${ }^{21}$.

\section{A quantitative model of Carbon concentration in diatoms: CFM-CC}

201 Our subcellular localization analysis suggests that proton transport by rhodopsin acidifies the inner side of the outermost membrane of the plastid (hereafter "the middle space"). To quantitatively test the effect of the decreased $\mathrm{pH}$ in the middle space on $\mathrm{C}$ fixation, we developed a simple quantitative model of carbonate chemistry combined with membrane transport and C fixation (CFM-CC: Cell Flux Model of C Concentration) (Fig. 4 upper panel). Previously, a comprehensive model of $\mathrm{CO}_{2}$ concentration within diatoms was developed ${ }^{22}$. CFM-CC uses a conceptually similar structure to this model, focusing on more specific membrane layers, designed to test the effect of altered $\mathrm{pH}$ in the middle space. 
$211\left(\left[\mathrm{CO}_{2}\right]_{m}\right)$ strongly depend on the $\mathrm{pH}\left(\mathrm{pH}_{\mathrm{m}}\right)$, suggesting that proton pumping by

212 rhodopsin could enhance $\mathrm{C}$ fixation (Fig. 4 bottom panel). The calculation of $\mathrm{C}$

213 chemistry in the middle space shows that decreasing $\mathrm{pH}_{\mathrm{m}}$ favors higher $\left[\mathrm{CO}_{2}\right]_{m}$ at a

214 given DIC concentration (Fig. 4A) (here we used $993 \mu \mathrm{mol} \mathrm{L}^{-1} 23$ ). We note that the

215 potential leaking of $\mathrm{CO}_{2}$ to the cytosol may change the DIC in the middle space, but

216 here we use a constant DIC value, since such effect has not been experimentally shown

217 and is difficult to quantify due to unknown factors (e.g., the balance of DIC uptake and

$218 \mathrm{CO}_{2}$ leaking). At the reference point (here $\left.\mathrm{pH}_{\mathrm{m}}=7.59^{23}\right),\left[\mathrm{CO}_{2}\right]_{m}$ is $17 \mu \mathrm{mol} \mathrm{L}^{-1}[\mathrm{y} 007$

219 03], but it increases to 64,410 , and $870 \mu \mathrm{mol} \mathrm{L}^{-1}$ for $\mathrm{pH}_{\mathrm{m}}$ values of 7, 6, and 5,

220 respectively [y007 04] (Fig. 4A).

Due to the increased $\left[\mathrm{CO}_{2}\right]_{m}, \mathrm{CO}_{2}$ concentration in the plastid $\left[\mathrm{CO}_{2}\right] p$ also increases with lowered $\mathrm{pH}_{\mathrm{m}}$, but the level of increase depends on $V_{\max } / D$ (the ratio of maximum $\mathrm{C}$ fixation rate to the diffusion constant) (Fig. 4B). When $V_{\max } / D$ is small, diffusion of $\mathrm{CO}_{2}$ from the middle space to the plastid dominates the influence, resulting in $\left[\mathrm{CO}_{2}\right]_{p}$ similar to $\left[\mathrm{CO}_{2}\right]_{m}$. In contrast, when $V_{\max } / D$ is large, $\mathrm{CO}_{2}$ uptake dominates, and there is small influence of $\left[\mathrm{CO}_{2}\right]_{m}$ on $\left[\mathrm{CO}_{2}\right]_{p}$.

The rate of $\mathrm{C}$ fixation increases with lowered $\mathrm{pH}_{\mathrm{m}}$, since the increased $\left[\mathrm{CO}_{2}\right]_{\mathrm{m}}$ leads to faster transport of $\mathrm{CO}_{2}$ into the plastid (Fig. 4C). However, the magnitude of such increase depends on $V_{\max } / D$. The model shows that the effect of $\mathrm{pH}_{\mathrm{m}}$ on $\mathrm{C}$ fixation

230 is larger when $V_{\max } / D$ is large, because the rate of C fixation is more strongly affected

231 by $\left[\mathrm{CO}_{2}\right]_{m}$ [eq. 6], which is directly influenced by $\mathrm{pH}_{\mathrm{m}}$. However, when $V_{\max } / D$ is small, 232 the $\mathrm{C}$ fixation rate is more strongly influenced by the $\mathrm{C}$ uptake kinetics [eq. 4], which 233 saturates at relatively low $\left[\mathrm{CO}_{2}\right]_{p}\left(K\right.$ value of $\left.44 \mu \mathrm{mol} \mathrm{L}{ }^{-1}{ }^{24}\right)$. The increase in $\mathrm{C}$ fixation 234 ranges 2.1-3.8, 3.2-24.2 and 3.4-51.2 times when $\mathrm{pH}_{\mathrm{m}}$ decreases from 7.59 to $7,6,5$ 235 respectively [y008 13]. These results suggest two things. First, any $V_{\max } / D, \mathrm{pH}$ has a considerable effect on $\mathrm{C}$ uptake. Second, it would be highly favorable for the cells to

237 have high $V_{\max }$, relative to the diffusivity of $\mathrm{CO}_{2}$ across the membrane. This is most 
238 likely the case since when there are multiple membranes between the middle space and

239 plastid, which would lower the $D$ value ${ }^{25,26}$. Thus, this simple yet elegant system with

240 rhodopsin to manipulate the $\mathrm{pH}_{\mathrm{m}}$ can provide a powerful mechanism in $\mathrm{C}$ concentration

241 and thus support a high $\mathrm{C}$ fixation in some rhodopsin-containing diatoms, enabling

242 them to be more productive phytoplankton in the ocean.

243 Such a CCM based on $\mathrm{CO}_{2}$ diffusion (termed pump-leak type) is known to be a

244 possible mechanism by placing CAs in appropriate locations. For example,

245 Nannochloropsis oceanica (Ochrophyta), possessing the same four membrane-bound

246 complex plastids as found in diatoms, is thought to generate $\mathrm{CO}_{2}$ by placing CA in the

247 middle space ${ }^{27}$. Besides, centric diatom Chaetoceros gracilis is thought to use

248 externally placed CAs to allow $\mathrm{CO}_{2}$ to flow into the cell ${ }^{28}$. In contrast to the CA-based

249 model, acidification-based model was formerly proposed to facilitate $\mathrm{CO}_{2}$ fixation of

$250 \mathrm{RuBisCO}$ in the thylakoid lumen of plastids; $\mathrm{HCO}_{3}{ }^{-}$is converted into $\mathrm{CO}_{2}$ by

251 acidification by photosynthetic proton pumping into the thylakoid lumen ${ }^{29}$. Our model

252 further expands the model and proposes that the middle space acidified by proton-

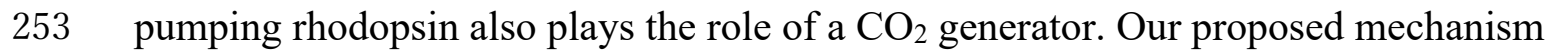

254 would be useful in most parts of the ocean where $\mathrm{CO}_{2}$ chronically limits photosynthesis,

255 but would be even more valuable in specific environments. For example, since CA,

256 which plays a central role in CCM, requires cobalt or zinc ions as the reaction center,

257 and photosynthetic proton pumping systems require iron, rhodopsin-derived acidic

258 pools may be useful for the transport of $\mathrm{Ci}$ in oceans where these metal ions are

259 depleted (such as the HNLC region of the North Pacific Ocean) ${ }^{30}$. Indeed, in the HNLC

260 region of the North Pacific, where the $P$. granii rhodopsin-containing cells were initially

261 isolated and sequenced ${ }^{31}$, it has been pointed out that primary production may be

262 limited by iron and influenced by other trace metals ${ }^{32}$. In other words, this mechanism

263 seems to be particularly effective in the ocean, where trace metals involved in CCM are

264 depleted. 


\section{Conclusion}

267 In this study, we have clarified the function and subcellular localization of PngR in a

268 photosynthetic diatom. Our results suggested that proton transport by rhodopsin creates

269 a local acidic pool inside the outermost membrane of the plastid. Based on the

270 quantitative simulation, we propose a conceptual model of rhodopsin-contributing CCM

271 (Extended Data Fig. 8). The acidic pool created by light is likely involved in the CCM

272 and provides positive feedback on carbon fixation efficiency. Future analyses of

273 cultured rhodopsin-bearing microbial eukaryotes would corroborate our results and

274 allow for the evaluation of how rhodopsin-mediated proton transport promote their

275 growth in the ocean.

276 


\section{References}

2781 Field, C. B., Behrenfeld, M. J., Randerson, J. T. \& Falkowski, P. Primary production of the

279 biosphere: Integrating terrestrial and oceanic components. Science 281, 237-240 (1998).

2802 Keeling, P. J. Diversity and evolutionary history of plastids and their hosts. Am. J. Bot. 91, 1481-1493 (2004).

2823 Marchetti, A., Catlett, D., Hopkinson, B. M., Ellis, K. \& Cassar, N. Marine diatom

283 proteorhodopsins and their potential role in coping with low iron availability. The ISME Journal 9, 2745-2748 (2015).

2854 Spudich, J. L., Yang, C. S., Jung, K. H. \& Spudich, E. N. Retinylidene proteins: Structures and functions from archaea to humans. Annu. Rev. Cell Dev. Biol. 16, 365-392 (2000). Oesterhelt, D. \& Stoeckenius, W. Rhodopsin-like protein from the purple membrane of Halobacterium halobium. Nature New Biol. 233, 149-152 (1971). Beja, O. et al. Bacterial rhodopsin: evidence for a new type of phototrophy in the sea. Science 289, 1902-1906 (2000).

2917 Sineshchekov, O. A., Jung, K. H. \& Spudich, J. L. Two rhodopsins mediate phototaxis to low- and high-intensity light in Chlamydomonas reinhardtii. Proc. Natl. Acad. Sci. U. S. A. 99, 8689-8694 (2002). Ernst, O. P. et al. Microbial and animal rhodopsins: structures, functions, and molecular mechanisms. Chem. Rev. 114, 126-163 (2014).

2969 Yoshizawa, S., Kawanabe, A., Ito, H., Kandori, H. \& Kogure, K. Diversity and functional analysis of proteorhodopsin in marine Flavobacteria. Environ. Microbiol. 14, 1240-1248 (2012).

$29910 \quad$ Kikuchi, M. et al. Functional expression of the eukaryotic proton pump rhodopsin OmR2 in Escherichia coli and its photochemical characterization. Sci. Rep. 11, 1-13 (2021).

11 Nagel, G. et al. Channelrhodopsin-1: A light-gated proton channel in green algae. Science 296, 2395-2398 (2002).

30312 Slamovits, C. H., Okamoto, N., Burri, L., James, E. R. \& Keeling, P. J. A bacterial proteorhodopsin proton pump in marine eukaryotes. Nat. Commun. 2 (2011).

30513 Balashov, S. P. et al. Xanthorhodopsin: a proton pump with a light-harvesting carotenoid antenna. Science 309, 2061-2064 (2005).

30714 Man, D. L. et al. Diversification and spectral tuning in marine proteorhodopsins. EMBO J. 22, 1725-1731 (2003).

30915 Miranda, M. R. M. et al. The photocycle and proton translocation pathway in a 310 cyanobacterial ion-pumping rhodopsin. Biophys. J. 96, 1471-1481 (2009).

$31116 \quad$ Nakajima, K., Tanaka, A. \& Matsuda, Y. SLC4 family transporters in a marine diatom 
(2013).

31417 Dorrell, R. G. et al. Principles of plastid reductive evolution illuminated by 315 nonphotosynthetic chrysophytes. Proc. Natl. Acad. Sci. U. S. A. 116, 6914-6923 (2019).

31618 Riebesell, U., Wolfgladrow, D. A. \& Smetacek, V. Carbon-dioxide limitation of marine317 phytoplankton growth-rates. Nature 361, 249-251 (1993).

31819 Tsuji, Y., Nakajima, K. \& Matsuda, Y. Molecular aspects of the biophysical CO2319 concentrating mechanism and its regulation in marine diatoms. J. Exp. Bot. 68, 3763-3772 (2017).

20 Matsuda, Y., Hopkinson, B. M., Nakajima, K., Dupont, C. L. \& Tsuji, Y. Mechanisms of carbon dioxide acquisition and $\mathrm{CO} 2$ sensing in marine diatoms: a gateway to carbon metabolism. Philos. Trans. R. Soc. B 372, 20160403 (2017).

Hopkinson, B. M., Dupont, C. L., Allen, A. E. \& Morel, F. M. M. Efficiency of the CO2concentrating mechanism of diatoms. Proc. Natl. Acad. Sci. U. S. A. 108, 3830-3837 (2011).

Hopkinson, B. M. A chloroplast pump model for the $\mathrm{CO} 2$ concentrating mechanism in the diatom Phaeodactylum tricornutum. Photosynth. Res. 121, 223-233 (2014). Burns, B. D. \& Beardall, J. Utilization of Inorganic Carbon by Marine Microalgae. J. Exp. Mar. Bio. Ecol. 107, 75-86 (1987). Jensen, E. L., Maberly, S. C. \& Gontero, B. Insights on the functions and ecophysiological relevance of the diverse carbonic anhydrases in microalgae. Int. J. Mol. Sci. 21, 2922 (2020).

Eichner, M. et al. N2 fixation in free - floating filaments of Trichodesmium is higher than in transiently suboxic colony microenvironments. New Phytol. 222, 852-863 (2019). Inomura, K., Wilson, S. T. \& Deutsch, C. Mechanistic model for the coexistence of nitrogen fixation and photosynthesis in marine Trichodesmium. mSystems 4, e0021000219 (2019). the carbon-concentrating mechanism in Nannochloropsis oceanica. Proc. Natl. Acad. Sci. U. S. A. 114, 4537-4542 (2017). (2021).

29 Raven, J. A. CO2-concentrating mechanisms: A direct role for thylakoid lumen acidification? Plant Cell Environ. 20, 147-154 (1997).

30 Moore, C. M. et al. Processes and patterns of oceanic nutrient limitation. Nat. Geosci. 6, 701-710 (2013). 
34931 Marchetti, A. et al. Comparative metatranscriptomics identifies molecular bases for the physiological responses of phytoplankton to varying iron availability. Proc. Natl. Acad. Sci. U. S. A. 109, E317-E325 (2012). (2008). 


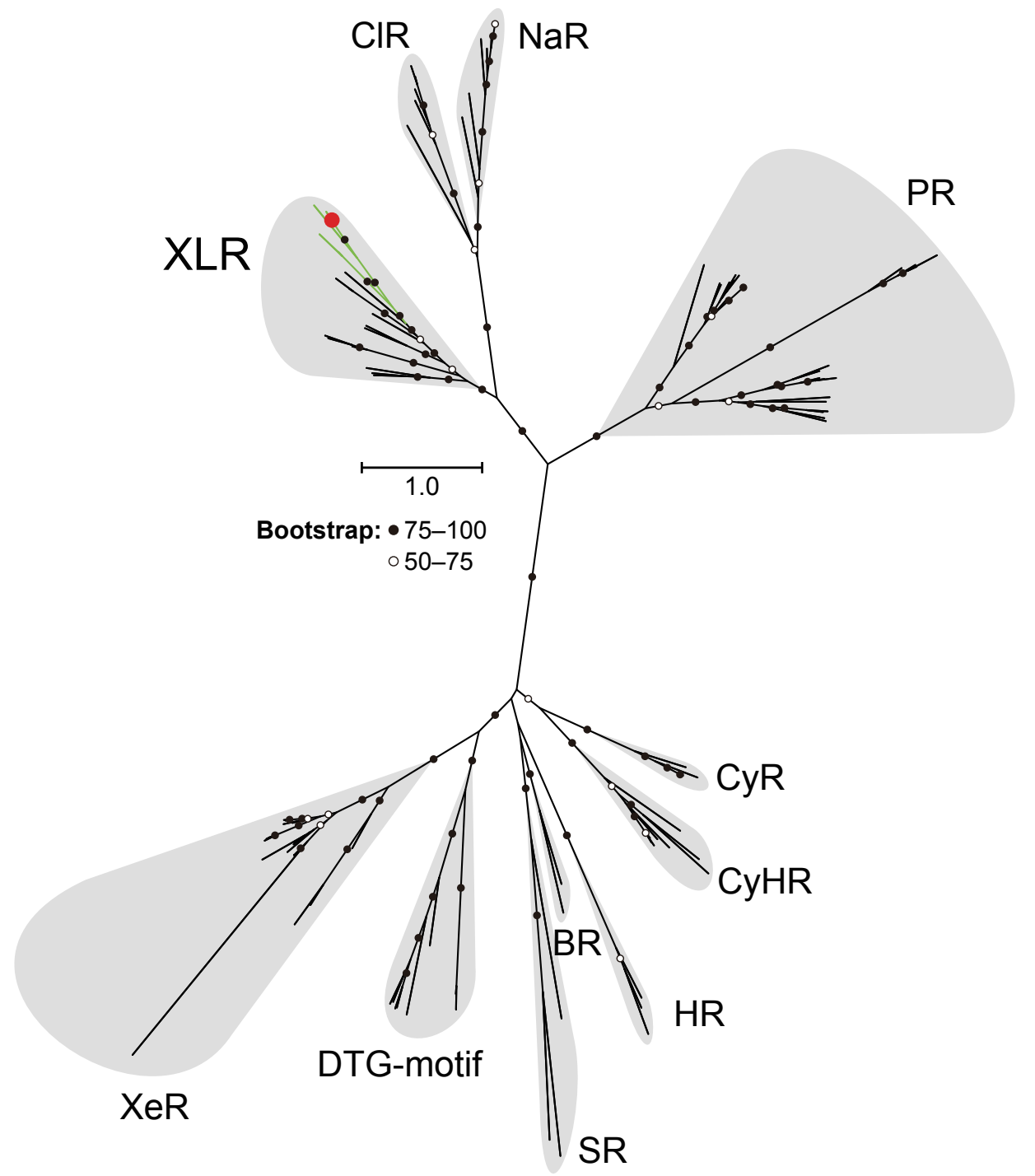

Figure 1. Yoshizawa et al.

357 Figure 1. Phylogenetic position of diatom rhodopsin. Maximum likelihood tree of amino acid sequences of microbial rhodopsins. Diatom rhodopsin (PngR) is indicated by a red circle and bootstrap probabilities $(\geq 50 \%)$ are indicated by black and white circles. Green branches indicate eukaryotic rhodopsins used in this analysis, and black branches indicate others. Rhodopsin clades are as follows: XLR (Xanthorhodopsin-like rhodopsin),

$362 \mathrm{ClR}$ ( $\mathrm{Cl}^{-}$-pumping rhodopsin), $\mathrm{NaR}\left(\mathrm{Na}^{+}\right.$-pumping rhodopsin), $\mathrm{PR}$ (proteorhodopsin),

363 XeR (xenorhodopsin), DTG-motif rhodopsin, SR (sensory rhodopsin-I and sensory 364 rhodopsin-II), BR (bacteriorhodopsin), HR (halorhodopsin), CyHR (cyanobacterial 365 halorhodopsin), and CyR (cyanorhodopsin). 


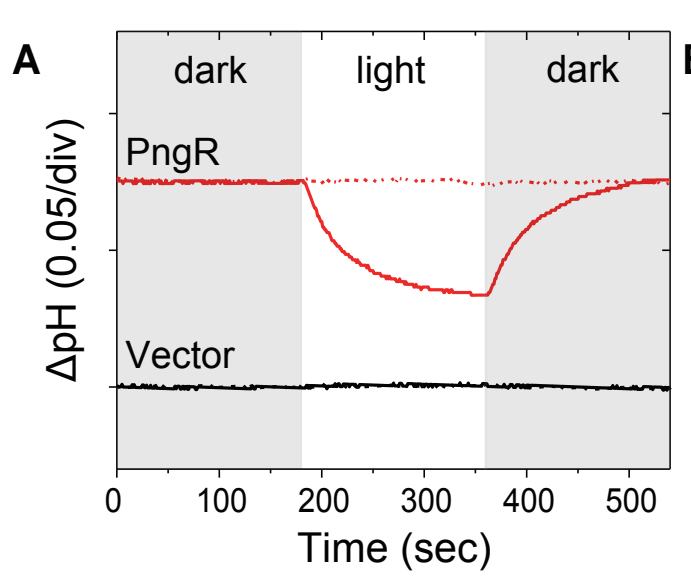

369

370

371

372

373

374

375

376 (w/v) DDM).

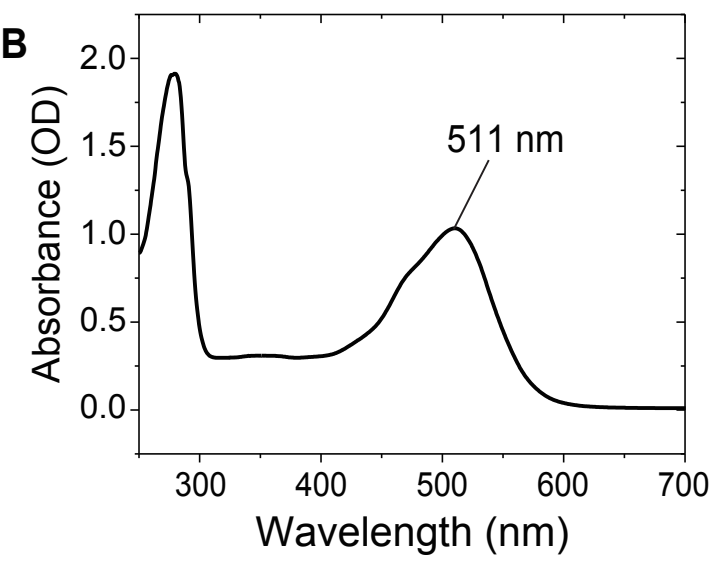

Figure 2. Yoshizawa et al.

\section{Figure 2. Light-induced pH changes and absorption spectrum of PngR. (A) Outward} proton pump activity of PngR in E. coli cells. Light-induced $\mathrm{pH}$ changes of solutions containing E. coli cells with the expression plasmid for PngR (upper panel) and the empty vector pET21a (lower panel) in the presence (red dashed line) or absence (red solid line) of CCCP. The white-filled region indicates the period of illumination. (B) Absorption spectrum of purified PngR in Buffer A (50 mM Tris- $\mathrm{HCl}, \mathrm{pH} 7.0,1 \mathrm{M} \mathrm{NaCl}$ and 0.05\% 
A
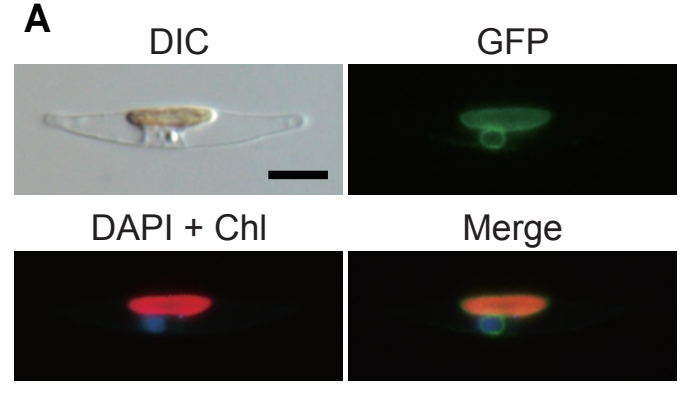

381

382

Figure 3. Subcellular localization of rhodopsins in diatom cell. (A) A transformed

383

384

385

386

387

388

389

390

391

392

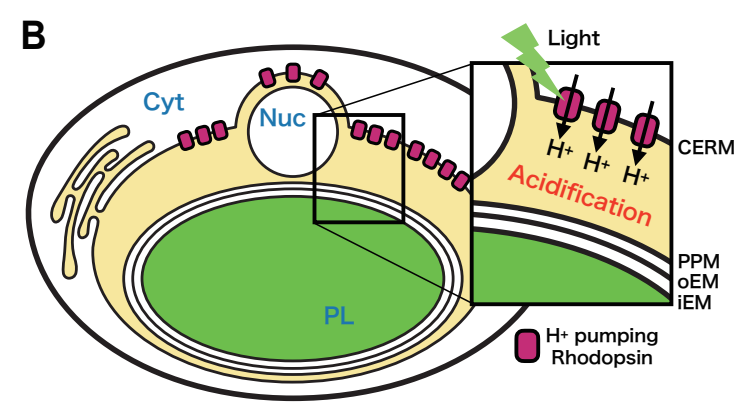

Figure 3. Yoshizawa et al. diatom cell was observed with DIC (Differential Interface Contrast) (Upper left). Green fluorescence from the recombinant PngR (GFP) (Upper right). The nuclear DNA stained with DAPI and the chlorophyll autofluorescence (DAPI $+\mathrm{Chl}$ ) and merged image (Merge) are shown in bottom left and bottom right, respectively. Scale bar indicates $5 \mu \mathrm{m}$. (B) A mode for subcellular localization of PngR. The proton transport of PngR acidifies the region (the middle space) surrounded by the membrane of CERM and PPM. Abbreviations are as follows: Cyt (Cytosol), Nuc (Nucleus), PL (Plastid), CERM (Chloroplast endoplasmic reticulum membrane), PPM (Periplastidial membrane), oEM (Outer plastid envelop membrane) and iEM (Internal plastid envelop membrane). 


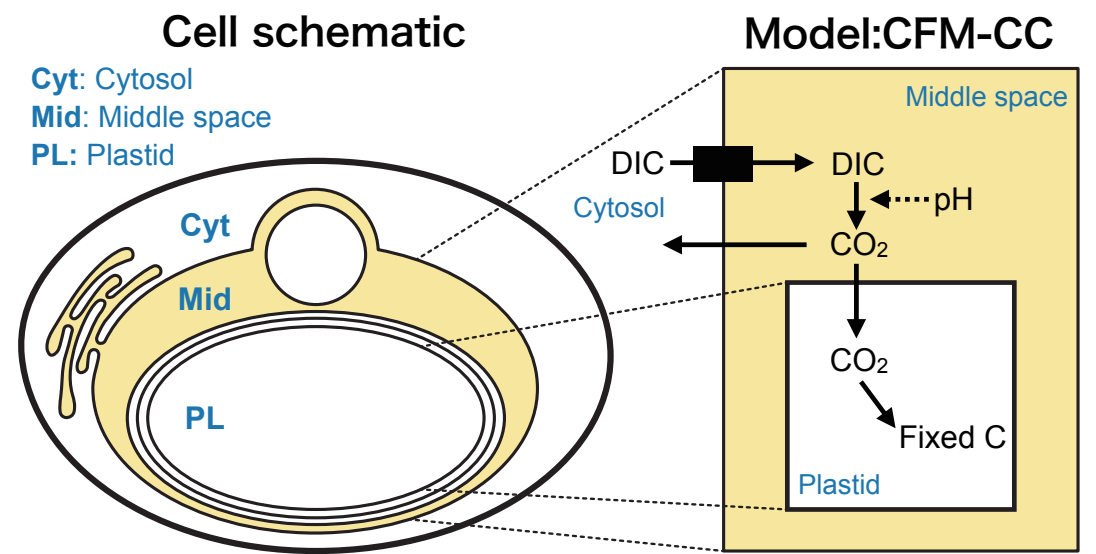

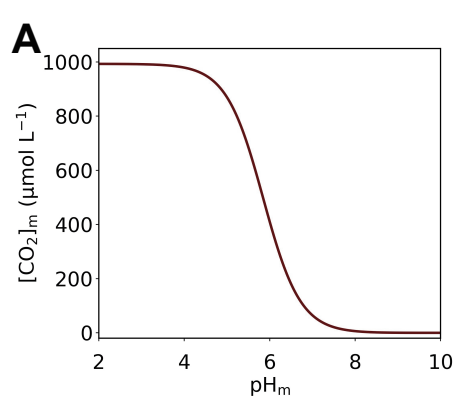

396

397

398

399

400

401

402

403

405

406 5 404 found for intracellular $\mathrm{pH}$ in a diatom ${ }^{23}$. (B) and $(\mathrm{C})$ are plotted for various $V_{\max } / D$. The
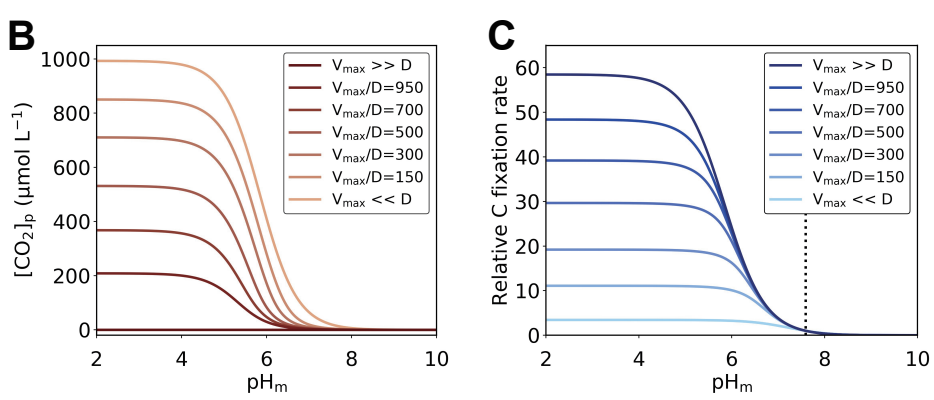

Figure 4. Yoshizawa et al.

Figure 4. A quantitative model of carbon concentration in diatoms. (Upper panel) Schematic of Cell Flux Model of C Concentration (CFM-CC). The left panel represents the actual cell and the right panel represents the model. Solid arrows show net flux of $\mathrm{C}$ and dashed arrow indicates the influence of $\mathrm{pH}$. (Bottom panel) The influence of $\mathrm{pH}$ in the middle space on $\mathrm{CO}_{2}$ concentrations and the photosynthesis rate. (A) $\mathrm{CO}_{2}$ concentrations in the middle space $\left[\mathrm{CO}_{2}\right]_{\mathrm{m}}$. (B) $\mathrm{CO}_{2}$ concentrations in the plastid $\left[\mathrm{CO}_{2}\right]_{\mathrm{p}}$.

403 (C) $\mathrm{C}$ fixation rate relative to that with $\mathrm{pH}$ in the middle space of 7.59, the only value we solution for $\left[\mathrm{CO}_{2}\right]_{\mathrm{m}}$ in $(\mathrm{A})$ is independent of $\operatorname{Vmax} / D$. 


\section{Rhodopsin sequences and phylogenetic analysis}

409 The rhodopsin sequence of the diatom Pseudo-nitzschia granii was previously

410 reported $^{3}$. All other rhodopsin sequences used in the phylogenetic analysis were

411 collected from the National Center for Biotechnology Information. Detailed information

412 on the strains used in this analysis is given in Extended Data Fig. 1. The sequences were

413 aligned using MAFFT version 7.453 with options'--genafpair' and '--maxiterate 1000'.

414 The phylogenetic tree was inferred using RAxML (v.8.2.12) with the

415 'PROTGAMMALGF' model using 1000 rapid bootstrap searches. Model selection was

416 performed with the ProteinModelSelection.pl script in the RAxML package. performed among protein sequences of Marine Microbial Eukaryote Transcriptome Sequencing Project (MMETSP) ${ }^{33}$. Namely, the phylogenetic placement of rhodopsin proteins from MMETSP using pplacer (v1.1.alpha19) ${ }^{34}$ was performed on a prebuilt large-scale phylogenetic tree of rhodopsins and extracted placements on the XLR clade using gappa (v0.6.0) $)^{35}$.

Gene preparation, protein expression and ion transport measurements of $E$. coli cells.

The full-length cDNA for PngR, whose codons were optimized for E. coli, were chemically synthesized by Eurofins Genomics and inserted into the NdeI-XhoI site of the $\mathrm{pET} 21 \mathrm{a}(+)$ vector as previously described ${ }^{36}$. A hexa-histidine-tag was fused at the C-terminus of PngR, which was utilized for purification of the expressed protein. The heterologous protein expression method is the same as previously reported ${ }^{37}$. E. coli BL21(DE3) cells harboring the cognate plasmid were grown at $37^{\circ} \mathrm{C}$ in LB medium supplemented with ampicillin (final concentration $=50 \mu \mathrm{g} \mathrm{mL}^{-1}$ ). Protein expression was induced at an optical density at $600 \mathrm{~nm}$ of $0.7-1.2$ with $1 \mathrm{mM}$ isopropyl $\beta$-d-1thiogalactopyranoside (IPTG) and $10 \mu \mathrm{M}$ all-trans retinal, after which the cells were 
434 incubated at $37^{\circ} \mathrm{C}$ for $3 \mathrm{~h}$. The proton transport activity of PngR was measured as light-

435 induced $\mathrm{pH}$ changes of suspensions of $E$. coli cells as previously described ${ }^{37}$. In short,

436 cells expressing PngR were washed more than three times in $150 \mathrm{mM} \mathrm{NaCl}$ and were

437 then resuspended in the same solution for measurements. Each cell suspension was

438 placed in the dark for several min and then illuminated using a $300 \mathrm{~W}$ Xenon lamp (ca.

$43930 \mathrm{~mW} \mathrm{~cm}{ }^{-2}$, MAX-303, Asahi spectra, Japan) through a $>460 \mathrm{~nm}$ long-pass filter

440 (Y48, HOYA, Japan) for $3 \mathrm{~min}$. Measurements were repeated under the same conditions

441 after the addition of the protonophore carbonyl cyanide m-chlorophenylhydrazone

$442(\mathrm{CCCP})($ final concentration $=10 \mu \mathrm{M})$. Light-induced $\mathrm{pH}$ changes were monitored

443 using a Horiba F-72 pH meter. All measurements were conducted at $25^{\circ} \mathrm{C}$ using a

444 thermostat (Eyela NCB-1200, Tokyo Rikakikai Co. Ltd, Japan).

Purification of PngR from $E$. coli cells and spectroscopic measurements of the purified rhodopsin.

E. coli cells expressing PngR were disrupted by sonication for $30 \mathrm{~min}$ in ice-cold water in a buffer containing $50 \mathrm{mM}$ Tris- $\mathrm{HCl}(\mathrm{pH} 7.0)$ and $300 \mathrm{mM} \mathrm{NaCl}$. The crude membrane fraction was collected by ultracentrifugation and solubilized with $1.0 \%(\mathrm{w} / \mathrm{v})$ n-dodecyl- $\beta$-d-maltoside (DDM, DOJINDO Laboratories, Japan). The solubilized fraction was purified by $\mathrm{Ni}^{2+}$ affinity column chromatography with a linear gradient of imidazole as described previously ${ }^{38}$. The purified protein was concentrated by centrifugation using an Amicon Ultra filter (30,000 $\mathrm{M}_{\mathrm{w}}$ cut-off; Millipore, USA). The sample media was then replaced with Buffer A (50 mM Tris-HCl, pH 7.0, $1 \mathrm{M} \mathrm{NaCl}$ and $0.05 \%(\mathrm{w} / \mathrm{v}) \mathrm{DDM})$ by ultrafiltration for 3-times.

Absorption spectra of purified proteins were recorded using a UV-2450 spectrophotometer (Shimadzu, Japan) at room temperature in Buffer A. The retinal composition in PngR was analyzed by high-performance liquid chromatography

460 (HPLC) as described previously ${ }^{39}$. For dark-adaptation, the samples were kept in the

461 dark condition for more than $72 \mathrm{hrs}$ at $4{ }^{\circ} \mathrm{C}$. For light-adaptation, the samples were 
462 illuminated for $3 \mathrm{~min}$ at $520 \pm 10 \mathrm{~nm}$, where the light power was adjusted to

463 approximately $10 \mathrm{~mW} \mathrm{~cm}{ }^{-2}$. The molar compositions of the retinal isomers were

464 calculated from the areas of the peaks in HPLC patterns monitored at $360 \mathrm{~nm}$ using the

465 extinction coefficients of retinal oxime isomers as described previously ${ }^{39}$. For $\mathrm{pH}$

466 titration experiments, the samples were suspended in Buffer $\mathrm{A}$. The $\mathrm{pH}$ values of the

467 samples were adjusted to the desired acidic values by adding $\mathrm{HCl}$, after which the

468 absorption spectra were measured at each $\mathrm{pH}$ value. All measurements were conducted

469 at room temperature (approx. $25^{\circ} \mathrm{C}$ ) under room light. After the measurements, the

470 reversibility of the spectral changes was checked to confirm that the sample was not

471 denatured during the measurements. The absorption changes at specific wavelengths

472 were plotted against $\mathrm{pH}$ values and the plots were fitted to the Henderson-Hasselbalch

473 equation assuming single $\mathrm{p} K_{\mathrm{a}}$ value as previously described ${ }^{37}$.

$700 \mathrm{~nm}$ at $5 \mathrm{~nm}$ intervals were obtained using a homemade computer-controlled flash photolysis system equipped with an Nd: YAG laser as an actinic light source. Using an optical parametric oscillator, the wavelength of the actinic pulse was tuned at $510 \mathrm{~nm}$ for PngR. The pulse intensity was adjusted to $2 \mathrm{~mJ}$ per pulse. All data were averaged to improve the signal-to-noise ratio $(\mathrm{n}=30)$. All measurements were conducted at $25^{\circ} \mathrm{C}$. For these experiments, the samples were suspended in Buffer A. After the measurements, the reproducibility of the data was checked to confirm that the sample was not denatured during the measurements. To investigate proton uptake and release during the photocycle, we used the $\mathrm{pH}$ indicator pyranine (final concentration $=100$ $\mu \mathrm{M}$, Tokyo Chemical Industry Co., Ltd, Japan), which has been extensively used to monitor light-induced $\mathrm{pH}$ changes in various rhodopsins. The $\mathrm{pH}$ changes in the bulk environment were measured as the absorption changes of pyranine at $450 \mathrm{~nm}$. The absorption changes of pyranine were obtained by subtracting the absorption changes of samples without pyranine from those of samples with pyranine. The experiments using pyranine were performed in an unbuffered solution containing $1 \mathrm{M} \mathrm{NaCl}$ and $0.05 \%$ 
(w/v) DDM ( $\mathrm{pH} 7.0)$ to enhance the signals. The results of 1000-traces were averaged

to improve the signal-to-noise ratio.

\section{Subcellular localization of PngR in the model diatom.}

494 The PngR:eGFP recombinant gene, coding the full length of PngR C-terminally tagged with eGFP, was cloned into the expression vector for the model diatom Phaeodactylum tricornutum, pPha-NR ${ }^{40}$, by CloneEZ (GenScript) following the manufacturer's instruction. The plasmid was electroporated into P. tricornutum cells with NEPA21 Super Electroporator (NEPAGENE), and transformed cells were selected with a Zeocin-based antibiotic treatment as described previously ${ }^{17,41}$. Selected clones were observed under an CCD color camera (Olympus). The nucleus stained with DAPI and chlorophyll autofluorescence from the plastid were observed with a $420 \mathrm{~nm}$ filter by 330 to $385 \mathrm{~nm}$ excitation. GFP fluorescence was detected with a 510 to $550 \mathrm{~nm}$ filter by 470 to $495 \mathrm{~nm}$ excitation.

\section{Quantitative model of C concentration in a diatom: CFM-CC}

507 Membrane transport model. In this model, we combined membrane transport of $\mathrm{CO}_{2}$ and $\mathrm{C}$ fixation (Fig. 4). Parameter definitions, units and values are provided in Supporting Information Table S2 and S3, respectively. The key model equation is the balance of $\mathrm{CO}_{2}$ concentration in cytosol $\left[\mathrm{CO}_{2}\right]_{p}$ :

$$
\frac{d\left[\mathrm{CO}_{2}\right]_{p}}{d t}=D\left(\left[\mathrm{CO}_{2}\right]_{m}-\left[\mathrm{CO}_{2}\right]_{p}\right)-V_{C f i x}
$$

511 where $t$ is time (units), $D$ is diffusion coefficient, $\left[\mathrm{CO}_{2}\right]_{m}$ is $\mathrm{CO}_{2}$ concentration (units) 512 in the middle space. Here the first term represents the diffusion of $\mathrm{CO}_{2}$ from the middle 513 space to cytosol and the second term $V_{C \text { fix }}$ represents the C fixation rate following 514 Michaelis-Menten kinetics ${ }^{22,42}$ :

$$
V_{C f i x}=V_{\max } \frac{\left[\mathrm{CO}_{2}\right]_{p}}{\left[\mathrm{CO}_{2}\right]_{p}+K}
$$

515 where $V_{\text {max }}$ is maximum $\mathrm{CO}_{2}$ fixation rate (units), and $K$ is a half saturation constant 516 (units). $\left[\mathrm{CO}_{2}\right]_{m}$ is obtained based on the carbonate chemistry in the middle space (see 
517 below). Under the steady-state, [eq. 1] with [eq. 2] becomes the following quadratic

518 relationship for $\left[\mathrm{CO}_{2}\right]_{p}$ :

$$
\left[\mathrm{CO}_{2}\right]_{p}^{2}+\left(\frac{V_{\max }}{D}+\mathrm{K}-\left[\mathrm{CO}_{2}\right]_{m}\right)\left[\mathrm{CO}_{2}\right]_{p}-K\left[\mathrm{CO}_{2}\right]_{m}=0 \quad \text { [eq. 3] }
$$

519 Solving this equation for $\left[\mathrm{CO}_{2}\right]_{p}^{2}$ leads to:

$$
\left[\mathrm{CO}_{2}\right]_{p}=\frac{-\left(\frac{V_{\max }}{D}+K-\left[\mathrm{CO}_{2}\right]_{m}\right)+\sqrt{\left(\frac{V_{\max }}{D}+K-\left[\mathrm{CO}_{2}\right]_{m}\right)^{2}+4 K}}{2} \quad \text { [eq. 4] }
$$

520 Note that the other solution for the negative rout is unrealistic since it may lead to the 521 overall negative value of $\left[\mathrm{CO}_{2}\right]_{p}$. Once we obtain $\left[\mathrm{CO}_{2}\right]_{p}$, we can then calculate the 522 rate of $C$ fixation $V_{C f i x}$ with [eq. 2].

Also, from [eq. 4], we can get two extreme solutions. First, when $V_{\max } \ll D$ (i.e., when the $\mathrm{CO}_{2}$ uptake capacity is small relative to the speed of $\mathrm{CO}_{2}$ diffusion) [eq. 4] leads to

$$
\left[\mathrm{CO}_{2}\right]_{p} \sim\left[\mathrm{CO}_{2}\right]_{m}
$$

526 With this relationship and [eq. 2], $V_{C f i x}$ is computed as follows:

$$
V_{C f i x} \sim V_{\max } \frac{\left[\mathrm{CO}_{2}\right]_{m}}{\left[\mathrm{CO}_{2}\right]_{m}+K}
$$

527

Second, when $V_{\max } \gg D$ (i.e., when the $\mathrm{CO}_{2}$ uptake capacity is high relative to the $\mathrm{CO}_{2}$ diffusion across the membrane) [eq. 4] becomes

$$
\left[\mathrm{CO}_{2}\right]_{p} \sim 0
$$

Under the steady state, [eq. 1] becomes

$$
V_{C f i x}=D\left(\left[\mathrm{CO}_{2}\right]_{m}-\left[\mathrm{CO}_{2}\right]_{p}\right)
$$

and plugging [eq. 7] into [eq. 8] leads to

$$
V_{C f i x} \sim D\left[\mathrm{CO}_{2}\right]_{m}
$$

531 and $V_{C f i x}$ is calculated. We note that $\left[\mathrm{CO}_{2}\right]_{p}>\left[\mathrm{CO}_{2}\right]_{m}$ could occur if there were membrane-bound transporters for $\mathrm{HCO}_{3}{ }^{-}$located on each membrane between the middle space and plastid ${ }^{22}$. However, such a set of transporters have not been discovered ${ }^{20}$. Thus, our model is fidelity to the current state of knowledge. Even if $\left[\mathrm{CO}_{2}\right]_{p}>\left[\mathrm{CO}_{2}\right]_{m}$ was the case, moderately decreased $\mathrm{pH}_{\mathrm{m}}$ and thus increased $\left[\mathrm{CO}_{2}\right]_{m}$ could be useful since they would decrease the gradient of $\mathrm{CO}_{2}$ across the membranes (i.e., $\left[\mathrm{CO}_{2}\right]_{p}$ vs $\left.\left[\mathrm{CO}_{2}\right]_{m}\right)$, mitigating the diffusive loss of $\mathrm{CO}_{2}$ from the plastid. 
540 obtain $\left[\mathrm{CO}_{2}\right]_{m}$. The model uses a given DIC (dissolved inorganic $\mathrm{C}$ ) concentration in the 541 middle space $[D I C]_{m}$ to calculate $\left[\mathrm{CO}_{2}\right]_{m}$ following the established equations for 542 carbon chemistry ${ }^{43}$.

$$
\left[\mathrm{CO}_{2}\right]_{m}=\frac{[\mathrm{DIC}]_{m}}{1+\frac{K_{1}}{\left[H^{+}\right]_{m}}+\frac{K_{1} K_{2}}{\left[H^{+}\right]_{m}^{2}}}
$$

543 where $\left[H^{+}\right]_{m}$ is $\mathrm{H}^{+}$concentration (thus $10^{-\mathrm{pH}} \mathrm{mol} \mathrm{l}^{-1}$ ) in the middle space and $K_{1}$ and $K_{2}$ 544 are temperature and salinity dependent parameters ${ }^{43,44}$ :

$$
\begin{aligned}
& K_{1}=10^{-p K_{1}} \\
& K_{2}=10^{-p K_{2}}
\end{aligned}
$$

545 where

$$
\begin{aligned}
p K_{1}=\frac{3633.86}{T} & -61.2172+9.6777 \ln (T)-0.011555 S \\
+ & 0.0001152 S^{2} \\
p K_{2}=\frac{471.78}{T}+ & 25.9290-3.16967 \ln (T)-0.01781 S \\
+ & 0.0001122 S^{2}
\end{aligned}
$$

546

547

Code availability

548 Code for CFM-CC is freely available from GitHub/Zenodo at 549 https://zenodo.org/record/5182712 (DOI: 10.5281/zenodo.5182712). 


\section{References}

33 Keeling, P. J. et al. The Marine Microbial Eukaryote Transcriptome Sequencing Project (MMETSP): Illuminating the Functional Diversity of Eukaryotic Life in the Oceans through Transcriptome Sequencing. PLoS Biol. 12, e1001889 (2014).

$55434 \quad$ Matsen, F. A., Kodner, R. B. \& Armbrust, E. V. pplacer: linear time maximum-likelihood and Bayesian phylogenetic placement of sequences onto a fixed reference tree. $B M C$ bioinformatics 11, 1-16 (2010).

Czech, L., Barbera, P. \& Stamatakis, A. Genesis and Gappa: processing, analyzing and visualizing phylogenetic (placement) data. Bioinformatics 36, 3263-3265 (2020). Hasegawa, M. et al. A unique clade of light-driven proton-pumping rhodopsins evolved in the cyanobacterial lineage. Sci. Rep. 10:16752 (2020). Inoue, S. et al. Spectroscopic characteristics of Rubricoccus marinus xenorhodopsin (RmXeR) and a putative model for its inward $\mathrm{H}+$ transport mechanism. Phys. Chem. Chem. Phys. 20, 3172-3183 (2018).

Kojima, K. et al. Vectorial Proton Transport Mechanism of RxR, a Phylogenetically Distinct and Thermally Stable Microbial Rhodopsin. Sci. Rep. 10:282 (2020).

Kojima, K. et al. Green-sensitive, long-lived, step-functional anion channelrhodopsin-2 variant as a high-potential neural silencing tool. J. Phys. Chem. 11, 6214-6218 (2020). Stork, S. et al. Distribution of the SELMA Translocon in Secondary Plastids of Red Algal Origin and Predicted Uncoupling of Ubiquitin-Dependent Translocation from Degradation. Eukaryot. Cell 11, 1472-1481 (2012). Miyahara, M., Aoi, M., Inoue-Kashino, N., Kashino, Y. \& Ifuku, K. Highly Efficient Transformation of the Diatom Phaeodactylum tricornutum by Multi-Pulse Electroporation. Biosci. Biotechnol. Biochem. 77, 874-876 (2013).

Berg, J., Tymoczko, J. \& Stryer, L. Biochemistry, ed 7th edition. (New York, WH Freeman, 2010).

57643 Emerson, S. \& Hedges, J. Chemical oceanography and the marine carbon cycle. (Cambridge University Press, 2008).

57844 Lueker, T. J., Dickson, A. G. \& Keeling, C. D. Ocean pCO2 calculated from dissolved inorganic carbon, alkalinity, and equations for K1 and K2: validation based on laboratory 


\section{Acknowledgements}

584 We thank Yusuke Matsuda for useful discussion. This work was supported by JSPS KAKENHI Grant

585 Numbers $18 \mathrm{~K} 19224$ and $18 \mathrm{H} 04136$ to S.Y., 19H04727, 21H00404 and 21H02446 to Y.S., and

58619 H03274 to R.K., and NSF grans OPP1745036 to A.M. This research was partially supported by the

587 Interdisciplinary Collaborative Research Program of the Atmosphere and Ocean Research Institute,

588 the University of Tokyo.

589

590 Author contributions

591 S.Y., A.M., Y.S., and R.K. designed the research; S.Y., T.A., K.K., K.Inomura. M.H., Y.N., M.K., 592 K.Ifuku. and R.K. performed the research; S.Y., K.K., K.Inomura., Y.N., M.K., G.A., H.M.,T.Y., H.F., 593 A.M., Y.S. and R.K. analyzed the data; and S.Y., K.Inomura., Y.S., and R.K. wrote the paper.

594

595 Competing interests

596 The authors declare no competing interests.

597

$598 \quad$ Additional information

599 Supplementary information is available for this paper.

600

601 Correspondence and requests for materials should be addressed to S.Y.

602 